\title{
Solvability of a Plane Elliptic Problem for the Flow in a Channel with a Surface-Piercing Obstacle
}

\author{
S. Gatti and D. Pierotti
}

\begin{abstract}
Let us consider the three-dimensional problem of steady flow of a heavy ideal fluid past a surface-piercing obstacle in a rectangular channel of constant depth. The flow is parallel at infinity upstream with constant velocity $c$. We discuss an approximate linear problem obtained in the limit of a "flat obstacle". This is a boundary value problem for the Laplace equation in a three-dimensional unbounded domain, with a second order condition on part of the boundary - the Neumann-Kelvin condition. By a Fourier expansion of the potential function we reduce the three-dimensional problem to a sequence of plane problems for the Fourier coefficients. For every value of the velocity $c$ these problems can be described in terms of a two parameter elliptic problem in a strip. We discuss a well-posed formulation of such problem by a special variational approach, relying on some a priori properties of finite energy solutions. As a result, we prove unique solvability for $c \neq c_{m, k}$, where $c_{m, k}$ is a known sequence of values depending on the dimensions of the channel and on the limit length of the obstacle. Accordingly, we can prove the existence of a solution of the three-dimensional problem; the related flow has in general a non-trivial wave pattern at infinity downstream. We also investigate the regularity of the solution in a neighborhood of the obstacle. The meaning of the singular values $c_{m, k}$ is discussed from the point of view of the nonlinear theory.
\end{abstract}

Keywords: Three-dimensional flow, Neumann-Kelvin condition, variational problems

AMS subject classification: 35J20, 35J25, 76B15

\section{Introduction}

Let us consider an infinitely long channel of constant depth $H$ and with rectangular cross section of constant width $L$, filled with a heavy, incompressible inviscid fluid. Assuming a constant, parallel flow with velocity $c$ at infinity upstream, we want to study the perturbed stationary flow due to the presence of a surface-piercing obstacle in the channel. The solution of this problem is a difficult task, since one has to satisfy a nonlinear condition (the Bernoulli condition) on a free boundary (the free surface of the fluid). Moreover, one must also determine the lines of contact between free surface and the obstacle's surface. Therefore, there are not many results in the mathematical literature concerning problems of this kind, despite their practical interest. Besides,

S. Gatti: Dip. di Matem., Univ. di Ferrara, via Machiavelli 35, 44100 Ferrara, Italy

D. Pierotti: Dip. di Matem. del Politecnico, Piazza L. da Vinci 32, 20133 Milano, Italy

ISSN 0232-2064 / \$2.50 (C) Heldermann Verlag Berlin 
most of such results are restricted to the two-dimensional case. This is true even in the framework of approximate linear theories, which avoid the difficulties related to the free boundary $[6,11,16]$. In the present work, we will consider a linearization of the problem of the flow past an obstacle in a channel, relying on the following assumptions:

(a) The obstacle has the same width of the channel's section, so that the free surface of the fluid is divided into two disconnected parts.

(b) The obstacle is "flat" in the sense that the thickness of its piercing part is small compared to the channel's dimensions.

Then we introduce a small positive parameter $\varepsilon$ such that for $\varepsilon \rightarrow 0$ the obstacle becomes a plane rectangular domain parallel to the unperturbed flow. It is shown that, for $\varepsilon=0$, the constant parallel flow is a solution of the problem. By assuming that the relevant quantities admit a (fractional) power expansion in $\varepsilon$, we linearize the problem around the solution at $\varepsilon=0$. In this way we obtain a three-dimensional boundary value problem in an unbounded domain for a harmonic function describing the perturbed velocity potential of the flow. Assuming that the solution can be represented by a suitable Fourier expansion, we reduce the three-dimensional linear problem to a sequence of boundary value elliptic problems in a two-dimensional strip for the Fourier coefficients. The main difficulty in dealing with these problems is related to the presence of a second order condition, the Neumann-Kelvin condition, on part of the boundary. In this case, as shown in [6 - 8], it is necessary to introduce two additional conditions, depending on two real parameters, for the existence of a unique solution. We discuss a particular form of these conditions and prove uniqueness and regularity theorems for the corresponding formulation of the problem. In particular, we prove unique solvability for every value of the velocity at infinity (upstream) with the exception of a known sequence, depending on the dimensions of the channel and on the length of the obstacle; the solution has (in general) oscillations at infinity downstream. Similar results has been obtained in [7, 8] for the plane Neumann-Kelvin problem with different kinds of additional conditions. Moreover, we show that for smooth enough obstacle's shapes the solution is continuous up to the boundary of the strip and that the trace on the linearized free boundary has continuous derivative. Finally, we discuss the conjecture (supported by the results of [17]) that for a unique pair of values of the parameters in the additional conditions the gradient of the solution is continuous in the closed strip.

The paper is organized as follows: in Section 2 we write the equations for the threedimensional flow potential and describe the linearization procedure for the case of a flat obstacle; from the resulting linear problem (problem P) we deduce the sequence of two dimensional elliptic problems (for the Fourier coefficients of the potential) and provide a well-posed formulation for a generic problem in the sequence (problem $P_{\lambda, \nu}$ ).

In Section 3 we prove some a priori properties of the solutions of the two-dimensional problem. In particular, we show that in general there are non-trivial oscillations at infinity behind the obstacle. The properties of finite energy solutions are also considered. In the subsequent Sections 4 - 5 we prove theorems on the unique solvability and on the regularity of solutions, starting from the discussion of a variational problem for finite energy solutions. Finally, in Section 6 we provide sufficient conditions for the solvability of the linearized three-dimensional problem and discuss the relevance of the results obtained for the nonlinear problem. 


\section{Statement of the problem}

We choose a reference frame such that the $x$-axis is directed as the unperturbed flow, the undisturbed free surface is represented by

$$
D_{0}=\{(x, y, z): x \in \mathbb{R}, 0 \leq y \leq L, z=0\}
$$

and the channel's bottom is the plane region

$$
\{(x, y, z): x \in \mathbb{R}, 0 \leq y \leq L, z=-H\}
$$

We assume that the surface of the obstacle is described by the equation

$$
z=\varepsilon f(x, y)
$$

where $\varepsilon>0$ is a small parameter and $f$ is a $C^{1}$ function defined in a domain $D_{f} \subset D_{0}$. We take

$$
D_{f}=\{(x, y, z): x \in J, 0 \leq y \leq L, z=0\}
$$

where $J \subset \mathbb{R}$ is an interval containing the origin. We further assume that $f$ is strictly negative for $x$ in some subinterval contained in $J$ (see Figures 1 and 2). The fluid surface has equation $z=h(x, y)$, where $h$ is an unknown function defined on a disconnected subset $D_{h} \subset D_{0}$.

Figure 1: Longitudinal section of the system fluid - obstacle

We set

$$
\begin{aligned}
D_{h}= & \left\{(x, y, z): 0 \leq y \leq L, x<g_{-}(y), z=0\right\} \\
& \cup\left\{(x, y, z): 0 \leq y \leq L, x>g_{+}(y), z=0\right\}
\end{aligned}
$$

where the two curves $x=g_{ \pm}(y) \quad(0 \leq y \leq L)$ represent the projection on the plane $z=0$ of the lines where the free surface meets the obstacle. Then, we have the conditions $g_{ \pm}(y) \in J, g_{-}(y)<0<g_{+}(y)$ and

$$
h\left(g_{ \pm}(y), y\right)=\varepsilon f\left(g_{ \pm}(y), y\right) \quad(0 \leq y \leq L)
$$


Figure 2: Channel and obstacle cross sections at $x=0$

The functions $g_{ \pm}$are also unknown and their determination is part of the problem. We denote by $\Omega$ the region filled with the fluid, i.e.

$$
\Omega=\left\{(x, y, z) \mid \begin{array}{l}
x \in \mathbb{R}, 0<y<L \\
-H<z<\varepsilon f(x, y) \text { if } g_{-}(y) \leq x \leq g_{+}(y) \\
-H<z<h(x, y) \text { if } x \leq g_{-}(y) \text { or } x \geq g_{+}(y)
\end{array}\right\} .
$$

Assuming the flow irrotational, we can state the problem in terms of a velocity potential $\Phi$, which must be a harmonic function in $\Omega$ by incompressibility. Further conditions on $\Phi$ follow by additional assumptions on the interaction of the fluid with the obstacle and with the channel's boundaries. In the case of a rigid obstacle, at every wetted point of its surface the velocity vector $\nabla \Phi$ lies in the tangent plane. Similarly, we require the vanishing of the components of $\nabla \Phi$ orthogonal to the channel's bottom and to the lateral walls, respectively. Moreover, it is known that the velocity field must be tangent at every (regular) point of the free surface. Finally, the height of a point on the free surface is related to the velocity field at the same point by the Bernoulli condition (see equation (2.5) below).

Then, the problem has the following form: find two real functions $g_{+}>0$ and $g_{-}<0$ in $[0, L]$, a real function $h$ in $D_{h}$ and a harmonic function $\Phi$ in $\Omega$ such that

$$
\begin{aligned}
\frac{1}{2}|\nabla \Phi(x, y, h(x, y))|^{2}+g h(x, y) & =\text { const } \quad \text { for }\left\{\begin{array}{l}
0<y<L \\
x<g_{-}(y) \text { or } x>g_{+}(y)
\end{array}\right. \\
\nabla \Phi(x, y, h(x, y)) \cdot \mathbf{n}_{h} & =0 \text { for }\left\{\begin{array}{l}
0<y<L \\
x<g_{-}(y) \text { or } x>g_{+}(y)
\end{array} \text { for } 0<y<L, g_{-}(y)<x<g_{+}(y)\right. \\
\nabla \Phi(x, y, \varepsilon f(x, y)) \cdot \mathbf{n}_{f} & =0 \\
\Phi_{y}(x, 0, z)=\Phi_{y}(x, L, z) & =0 \\
\Phi_{z}(x, y,-H) & =0 \\
\lim _{x \rightarrow-\infty} \nabla \Phi(x, y, z) & =c \mathbf{i} \\
\lim _{x \rightarrow-\infty} h(x, y) & =0
\end{aligned}
$$


where

$$
\begin{aligned}
& \mathbf{n}_{h}(x, y)=h_{x}(x, y) \mathbf{i}+h_{y}(x, y) \mathbf{j}-\mathbf{k} \\
& \mathbf{n}_{f}(x, y)=\varepsilon f_{x}(x, y) \mathbf{i}+\varepsilon f_{y}(x, y) \mathbf{j}-\mathbf{k} .
\end{aligned}
$$

Equations (2.5) - (2.6) are, respectively, the Bernoulli condition and the kinematic condition on the free surface. Equations (2.7) - (2.9) represent the previously discussed boundary conditions on the obstacle and on the channel's walls. Equations (2.10) (2.11) state that the fluid is unperturbed at infinity upstream. Finally, we recall the condition (2.3) connecting the free surface and the obstacle.

Taking account of the limit conditions (2.10) - (2.11) in relation (2.5) we find that the constant at the right-hand side is $\frac{1}{2} c^{2}$. As one can readily check, when $\varepsilon=0$ problem (2.5) - (2.11) has the solution

$$
\begin{gathered}
\Phi(x, y, z)=c x \\
h(x, y)=0
\end{gathered}
$$

so that in the limit case of a rectangular plate the problem is solved by the free parallel flow. For small enough $\varepsilon$ it is reasonable to look for small perturbations of the above solution. Then we introduce new variables $\varphi$ and $\tilde{h}$ through the relations

$$
\begin{aligned}
& \Phi=c x+\varepsilon \varphi \\
& h=\varepsilon \tilde{h} .
\end{aligned}
$$

Clearly, the function $\varphi$ has to satisfy

$$
\Delta \varphi=0
$$

in $\Omega$.

In order to get a well defined linearized problem, we must specify the limit for $\varepsilon \rightarrow 0$ of boundary conditions $(2.5)-(2.11)$. Let us first consider the particular case $f(x, y)=f_{0}(x)$ in condition $(2.7)$; this corresponds to an obstacle with longitudinal section independent of $y$. In this case it is reasonable to assume that a small perturbation of the free parallel flow is described by a solution independent of $y$, so that we can put $\varphi(x, y, z)=\varphi_{0}(x, z)$ and $\tilde{h}(x, y)=h_{0}(x)$ in (2.12). Consequently, the functions $g_{ \pm}$in relations $(2.2)-(2.7)$ will be equal to the (unknown) constants $x_{ \pm}$. Then, letting $\varepsilon \rightarrow 0$ and assuming that $x_{ \pm} \rightarrow \tilde{x}_{ \pm}$, we get from (2.5) - (2.7) the limit conditions

$$
\begin{aligned}
\partial_{x} \varphi_{0}(x, 0)+\frac{g}{c} h_{0}(x) & =0 & & \left(x>\tilde{x}_{+}, x<\tilde{x}_{-}\right) \\
\partial_{z} \varphi_{0}(x, 0) & =c h_{0}^{\prime}(x) & & \left(x>\tilde{x}_{+}, x<\tilde{x}_{-}\right) \\
\partial_{z} \varphi_{0}(x, 0) & =c f_{0}^{\prime}(x) & & \left(\tilde{x}_{-}<x<\tilde{x}_{+}\right) .
\end{aligned}
$$

These boundary conditions, involving the (two-dimensional) potential $\varphi_{0}$, are more conveniently written in terms of the harmonic conjugate field components $u_{0}=\partial_{x} \varphi_{0}$ and $v_{0}=\partial_{z} \varphi_{0}$ as

$$
\begin{aligned}
u_{0}(x, 0)+\frac{g}{c} h_{0}(x) & =0 & & \left(x>\tilde{x}_{+}, x<\tilde{x}_{-}\right) \\
v_{0}(x, 0) & =c h_{0}^{\prime}(x) & & \left(x>\tilde{x}_{+}, x<\tilde{x}_{-}\right) . \\
v_{0}(x, 0) & =c f_{0}^{\prime}(x) & & \left(\tilde{x}_{-}<x<\tilde{x}_{+}\right) .
\end{aligned}
$$


Moreover, differentiating (2.17) and using (2.18), we can eliminate the unknown function $h_{0}$ and obtain

$$
\partial_{x} u_{0}(x, 0)+\frac{g}{c^{2}} v_{0}(x, 0)=0 \quad\left(x>\tilde{x}_{+}, x<\tilde{x}_{-}\right) .
$$

By the Cauchy-Riemann equations, we now get the condition

$$
\partial_{z} v_{0}(x, 0)-\frac{g}{c^{2}} v_{0}(x, 0)=0 \quad\left(x>\tilde{x}_{+}, x<\tilde{x}_{-}\right) .
$$

Finally, from (2.9) - (2.11) we easily obtain the additional boundary condition

$$
v_{0}(x,-H)=0 \quad(x \in \mathbb{R})
$$

and the asymptotic conditions

$$
\lim _{x \rightarrow-\infty} u_{0}(x, z)=\lim _{x \rightarrow-\infty} v_{0}(x, z)=0 .
$$

Summing up the above discussion, the linearized problem in the case of an obstacle with constant longitudinal section amounts to find a harmonic function $v_{0}\left(=\partial_{z} \varphi_{0}\right)$ in a two-dimensional strip, satisfying boundary conditions (2.19) - (2.21) and asymptotic condition (2.22) (with its harmonic conjugate). The unique solvability of this problem have been stated in both the cases of supercritical (i.e. $c>\sqrt{g H}$ ) and subcritical (i.e. $c<\sqrt{g H}$ ) flow velocities (see [14]). By these results, the proof of the solvability of the two-dimensional nonlinear free boundary problem has been achieved (at least for the case $c>\sqrt{g H}$, see [12]). As a consequence, it can be proved that for every datum $\varepsilon f_{0}(x)$ in (2.7) (independent of $y$ and smooth enough in $x$ ) there exist two functions $\varphi_{0}=\varphi_{0}(x, z)$ and $h_{0}=h_{0}(x)$ and constants $x_{ \pm}$with the following properties:

1) For small enough $\varepsilon$, the set of four functions $c+\varepsilon \varphi_{0}, \varepsilon h_{0}, y \mapsto g_{ \pm}(y)=x_{ \pm}$ is a solution independent of $y$ of the nonlinear, free boundary problem $(2.5)-(2.11)$ with additional condition (2.3). Moreover, $\varphi_{0}$ is harmonic in a $\mathcal{C}^{1}$ domain (the upper boundary being a $\mathcal{C}^{1}$ streamline formed by the profiles of the obstacle $f_{0}$ and of the free boundary $h_{0}$, see [12]) and with continuous derivatives up to the boundary.

2) For $\varepsilon \rightarrow 0$, the values $x_{ \pm}$tend to finite limits $\tilde{x}_{ \pm} \in J$, with $\tilde{x}_{-}<0<\tilde{x}_{+}$, and $\varphi_{0}$ converges to a harmonic function in the two-dimensional strip $\mathbb{R} \times(-H, 0)$, satisfying the linear boundary value problem (2.19) - (2.21).

Let us now go back to the three-dimensional problem and make the further assumption that the function $f$ in (2.1) has the form:

$$
f(x, y)=f_{0}(x)+\varepsilon^{\alpha} \mathcal{F}(x, y)
$$

where $0<\alpha<1$ and $\mathcal{F}$ is defined in $D_{f}$ (see below equation (2.1)). Hence, it is reasonable to look for a perturbed field $\varphi$ which, in the limit $\varepsilon \rightarrow 0$, approaches a solution independent of $y$. This in turn justifies the assumption that the two functions $g_{ \pm}$in $(2.2)-(2.7)$ tend to finite constant limits for $\varepsilon \rightarrow 0$. More precisely, we will write for the perturbed field and the free boundary in $(2.12)$

$$
\begin{aligned}
\varphi(x, y, z) & =\varphi_{0}(x, z)+\varepsilon^{\alpha} \Psi(x, y, z) \\
\tilde{h}(x, y) & =h_{0}(x)+\varepsilon^{\alpha} \Xi(x, y)
\end{aligned}
$$


where $\varphi_{0}$ and $h_{0}$ satisfy properties 1 ) and 2). Then condition (2.3) is satisfied in the limit $\varepsilon \rightarrow 0$ if the two functions $g_{ \pm}$converge to the constant limits $\tilde{x}_{ \pm}$(depending on $\left.\varphi_{0}\right)$. Assuming this, let us consider conditions (2.5) - (2.7) up to the terms of order $\varepsilon^{1+\alpha}$. Letting $\varepsilon \rightarrow 0$ and taking account of $(2.14)-(2.16)$ we find that the function $\Psi$ is harmonic in the three-dimensional domain $\mathbb{R} \times(0, L) \times(-H, 0)$ and satisfies the boundary conditions

$$
\begin{aligned}
\Psi_{x}(x, y, 0)+\frac{g}{c} \Xi(x, y) & =0 & & \left(x<\tilde{x}_{-}, x>\tilde{x}_{+}, 0<y<L\right) \\
\Psi_{z}(x, y, 0) & =c \Xi_{x}(x, y) & & \left(x<\tilde{x}_{-}, x>\tilde{x}_{+}, 0<y<L\right) \\
\Psi_{z}(x, y, 0) & =c \mathcal{F}_{x}(x, y) & & \left(\tilde{x}_{-}<x<\tilde{x}_{+}, 0<y<L\right) .
\end{aligned}
$$

Note that, due to our choice of the exponent $\alpha$ in $(2.23)-(2.25)$, the limit problem for the perturbed potential $\Psi$ depends on the first order term $\varphi_{0}$ only through the two constants $\tilde{x}_{ \pm}$. We can get rid of the unknown function $\Xi_{x}$ differentiating (2.26) with respect to $x$ and using (2.27). Hence we obtain

$$
\Psi_{x x}(x, y, 0)+\frac{g}{c^{2}} \Psi_{z}(x, y, 0)=0 \quad\left(x>\tilde{x}_{+}, x<\tilde{x}_{-} ; 0<y<L\right) .
$$

The above relation is the well known Neumann-Kelvin condition for the (perturbed) velocity potential $\Psi[6-10]$.

Finally, again from (2.8) - (2.11) we get the additional boundary conditions

$$
\begin{aligned}
\Psi_{y}(x, 0, z)=\Psi_{y}(x, L, z) & =0 & & (x \in \mathbb{R},-H<z<0) \\
\Psi_{z}(x, y,-H) & =0 & & (x \in \mathbb{R}, 0<y<L)
\end{aligned}
$$

and the asymptotic condition

$$
\lim _{x \rightarrow-\infty} \nabla \Psi(x, y, z)=0 .
$$

Thus, the "flat obstacle linearization" of the flow equations lead us to the following boundary value problem:

Problem P. Find a harmonic function $\Psi$ in the three-dimensional domain $\Omega_{0}=$ $\mathbb{R} \times(0, L) \times(-H, 0)$ satisfying boundary conditions $(2.28)-(2.31)$ and asymptotic condition $(2.32)$.

The geometry of the domain $\Omega_{0}$ and conditions (2.30) lead us to represent a solution of problem $P$ by a Fourier expansion of the form

$$
\Psi(x, y, z)=\sum_{m=0}^{\infty} \psi_{m}(x, z) \cos \left(\frac{m \pi}{L} y\right)
$$

Correspondingly, we will write

$$
\mathcal{F}(x, y)=\sum_{m=0}^{\infty} \mathcal{F}_{m}(x) \cos \left(\frac{m \pi}{L} y\right)
$$


Then, assuming that the right sides of (2.33) - (2.34) are convergent and (two times) term-wise differentiable up to the boundary of $\Omega_{0}$, we find that the Fourier coefficients $\psi_{m}$ solve the two-dimensional problems

$$
\begin{array}{ll}
\Delta \psi_{m}(x, z)=\frac{m^{2} \pi^{2}}{L^{2}} \psi_{m}(x, z) & ((x, z) \in \mathbb{R} \times(-H, 0)) \\
\partial_{x x} \psi_{m}(x, 0)+\frac{g}{c^{2}} \partial_{z} \psi_{m}(x, 0)=0 & \left(x>\tilde{x}_{+}, x<\tilde{x}_{-}\right) \\
\partial_{z} \psi_{m}(x, 0)=c \mathcal{F}_{m}^{\prime}(x) & \left(\tilde{x}_{-}<x<\tilde{x}_{+}\right) \\
\partial_{z} \psi_{m}(x,-H)=0 & (x \in \mathbb{R}) \\
\lim _{x \rightarrow-\infty} \psi_{m}(x, z)=\lim _{x \rightarrow-\infty} \nabla \psi_{m}(x, z)=0
\end{array}
$$

with $m \geq 1$. For $m=0$, only the condition $\lim _{x \rightarrow-\infty} \nabla \psi_{0}(x, z)=0$ is required in (2.39); in this case, we have the same problem solved by $\varphi_{0}$. For $m \geq 1$ we have a boundary value problem in a two-dimensional strip for the elliptic operator $\left(-\Delta+\lambda_{m}\right)$ where $\lambda_{m}=\frac{m^{2} \pi^{2}}{L^{2}}$. As clarified in [6 - 8] for the Neumann-Kelvin problem, relations (2.35) - (2.39) must be supplemented with two conditions at the obstacle endpoints in order to get a well-posed problem; we will show later (see also [7]) that for smooth enough boundary data a solution of problem (2.35) - (2.39) is continuous at the points $\left( \pm x_{0}, 0\right)$. Then we can state:

Definition 2.1 We say that a function $\psi_{m}$ is a solution of problem $P_{\lambda_{m}} \quad(m \geq 1)$ if it satisfies conditions (2.35) - (2.39) and

$$
\psi_{m}\left(\tilde{x}_{ \pm}, 0\right)=\alpha_{ \pm}, \quad \alpha_{ \pm} \in \mathbb{R} .
$$

The role of (2.40) in connection with problem $\mathrm{P}$ above will be discussed in the last section.

Remark 2.1 Note that no additional conditions are required in problem $P_{0}$, since it can be solved in terms of the harmonic conjugate field components (see (2.19) - (2.22) and the subsequent discussion) avoiding the Neumann-Kelvin condition (2.36); in this way, one selects the "least singular potential" which has continuous derivatives up to the boundary.

In this paper we provide a precise statement of problem $P_{\lambda_{m}} \quad(m \geq 1)$ and discuss its unique solvability in a suitable functional space of Sobolev type; to this aim, some definitions and notations are in order. We first note that, possibly translating the origin, we can take $\tilde{x}_{ \pm}= \pm x_{0}$ in (2.36) - (2.37). Moreover, we will drop the index $m$ in all the equations and consider the problem for a generic $\lambda>0$ and with a Neumann datum $\mathcal{G}$. Finally, we put $\nu=\frac{g}{c^{2}}$.

Let us now denote the strip

$$
S_{H}=\left\{(x, z) \in \mathbb{R}^{2}:-H<z<0\right\} .
$$

We will call $B=\mathbb{R} \times\{-H\}$ the bottom. On the upper margin $\mathbb{R} \times\{0\}$ we denote by $I$ the open interval $\left(-x_{0}, x_{0}\right) \times\{0\}$ and set $F=\left\{\mathbb{R} \backslash\left[-x_{0}, x_{0}\right]\right\} \times\{0\}$. Then, for every fixed $\lambda>0$ and $\nu>0$, we consider the following problem. 
Problem $P_{\lambda, \nu}$. Given a function $\mathcal{G} \in L^{2}(I)$ and two real numbers $\alpha_{ \pm}$, find $\psi$ satisfying $\psi \in H^{1}(\mathcal{A})$ for every bounded domain $\mathcal{A} \subset S_{H}$, and such that

$$
\begin{array}{rlrl}
\Delta \psi & =\lambda \psi & & \text { in } S_{H} \\
\psi_{z}=\mathcal{G} & & \text { on } I \\
\psi_{x x}+\nu \psi_{z}=0 & & \text { on } F \\
\psi_{z}=0 & & \text { on } B .
\end{array}
$$

Moreover, the trace $\left.\psi\right|_{F}$ is continuous up to the points $\left( \pm x_{0}, 0\right)$ and

$$
\psi\left( \pm x_{0}, 0\right)=\alpha_{ \pm}
$$

Finally, $\psi$ fulfills the asymptotic conditions

$$
\lim _{x \rightarrow-\infty} \psi(x, z)=\lim _{x \rightarrow-\infty}|\nabla \psi(x, z)|=0
$$

uniformly with respect to $z \in[-H, 0]$ and

$$
\sup _{(x, z) \in S_{H} \backslash A}(|\psi(x, z)|+|\nabla \psi(x, z)|)<\infty
$$

where $A$ is any neighborhood of $I$.

We will discuss the solvability of the above problem for every positive value of $\lambda$ in (2.41) and of $\nu$ in (2.43). We remark that the solutions of problem $P_{\lambda, \nu}$ must be locally in $H^{1}$ and bounded together with their derivatives outside any neighborhood of the beam (inequality (2.47)). The first condition is necessary for the local finiteness of the kinetic energy of the perturbed potential (2.33). The latter one can be related to the boundedness of the velocity field $\nabla \Psi$, at least outside any neighborhood of the obstacle. Finally, note that we only require continuity of the trace on $F$ in order to give meaning to $(2.45)$.

\section{The two-dimensional problem: a priori properties of the solutions}

In the present section, we discuss some a priori properties of the solution to problem $P_{\lambda, \nu}$ which play a crucial role in the proof of solvability. To begin with, we shall consider in more detail the asymptotic properties. Let us denote with $\theta$ the characteristic function of the interval $(0,+\infty)$. Then we have

Proposition 3.1. Let $\psi \in H_{l o c}^{1}\left(S_{H}\right)$ be a solution to problem $P_{\lambda, \nu}$. Then, for any neighborhood $A$ of $I, \psi$ is smooth in $\overline{S_{H}} \backslash A$ and there are real constants $\mathcal{A}$ and $\mathcal{B}$ such that

$$
\sup _{\overline{S_{H} \backslash A}} e^{\sqrt{\lambda+\alpha_{1}^{2}}|x|}\left|\psi(x, z)-\theta(x)\left[\mathcal{A} \sin \left(\mu_{\lambda} x\right)+\mathcal{B} \cos \left(\mu_{\lambda} x\right)\right] \cosh \left[\nu_{\lambda}(z+H)\right]\right|<\infty
$$


where $\alpha_{1} \in\left(0, \frac{\pi}{2 H}\right)$ and $\nu_{\lambda}>\sqrt{\lambda}$ satisfy, respectively,

$$
\begin{aligned}
\tan \left(\alpha_{0} H\right) & =\frac{1}{\nu}\left(\alpha_{0}+\frac{\lambda}{\alpha_{0}}\right) \\
\tanh \left(\nu_{\lambda} H\right) & =\frac{1}{\nu}\left(\nu_{\lambda}-\frac{\lambda}{\nu_{\lambda}}\right)
\end{aligned}
$$

and

$$
\mu_{\lambda}=\sqrt{\nu_{\lambda}^{2}-\lambda}
$$

Proof. The smoothness properties of $\psi$ follow by standard regularity results for weak solutions of elliptic boundary value problems. Let us fix $R>x_{0}$ and solve equation (2.41) with boundary conditions $(2.43)-(2.44)$ by separation of variables in the regions $(R, \infty) \times(-H, 0)$ and $(-\infty,-R) \times(-H, 0)$. In this way, we will get series expansions for $\psi$ in the two regions (see [14]). By elementary calculations, we have the following eigenvalue problem (see also [3]) depending on the two parameters $\lambda$ and $\nu$ :

$$
\left.\begin{array}{rl}
-Z^{\prime \prime}(z) & =\kappa Z(z) \text { in }(-H, 0) \\
\lambda Z(0)+\nu Z^{\prime}(0) & =-\kappa Z(0) \\
Z^{\prime}(-H) & =0
\end{array}\right\} .
$$

It can be shown that this problem is self-adjoint (in a suitably defined Hilbert space, see Remark 3.1 below). We find a unique negative eigenvalue $\bar{\kappa}=-\nu_{\lambda}{ }^{2}<-\lambda$ where $\nu_{\lambda}$ is the unique (positive) solution of equation (3.3). The relative eigenspace is generated by the function

$$
\bar{Z}(z)=\cosh \left[\nu_{\lambda}(z+H)\right] .
$$

Moreover, there is a sequence of positive eigenvalues $\kappa_{n}=\alpha_{n}^{2}(n \geq 1)$ with

$$
\tan \left(\alpha_{n} H\right)=\frac{1}{\nu}\left(\alpha_{n}+\frac{\lambda}{\alpha_{n}}\right), \quad \alpha_{n} \in\left((n-1) \frac{\pi}{H},(2 n-1) \frac{\pi}{2 H}\right)
$$

whose eigenspaces are generated by the functions

$$
Z_{n}(z)=\cos \left[\alpha_{n}(z+H)\right] .
$$

Then, equations $(2.41),(2.43)-(2.44)$ are satisfied by the functions

$$
\begin{aligned}
& \gamma_{n}(x, z)=e^{ \pm \sqrt{\lambda+\alpha_{n}^{2}} x} \cos \left[\alpha_{n}(z+H)\right] \\
& \bar{\gamma}^{1}(x, z)=\cos \left(\mu_{\lambda} x\right) \cosh \left[\nu_{\lambda}(z+H)\right] \\
& \bar{\gamma}^{2}(x, z)=\sin \left(\mu_{\lambda} x\right) \cosh \left[\nu_{\lambda}(z+H)\right] .
\end{aligned}
$$

Taking account of asymptotic condition (2.46) - (2.47) we obtain the expansions

$$
\psi(x, z)= \begin{cases}{\left[\mathcal{A} \sin \left(\mu_{\lambda} x\right)+\mathcal{B} \cos \left(\mu_{\lambda} x\right)\right] \cosh \left[\nu_{\lambda}(z+H)\right]} & \\ +\sum_{n=1}^{\infty} a_{n} e^{-\sqrt{\lambda+\alpha_{n}^{2}} x} \cos \left[\alpha_{n}(z+H)\right] & \text { if } x>R \\ \sum_{n=1}^{\infty} b_{n} e^{\sqrt{\lambda+\alpha_{n}^{2}} x} \cos \left[\alpha_{n}(z+H)\right] & \text { if } x<-R\end{cases}
$$

where the coefficients $\mathcal{A}, \mathcal{B}$ and $a_{n}, b_{n}$ are uniquely determined by $\psi(R, \cdot)$ and $\psi(-R, \cdot)$ on $[-H, 0]$. Then the proposition follows 
Remark 3.1. Let us define the bilinear form

$$
(u, v)_{\lambda, \nu}=\int_{-H}^{0} u(z) v(z) d z-\frac{1}{\nu} u_{\lambda, \nu} v_{\lambda, \nu}
$$

in $L^{2}(-H, 0)$ where

$$
u_{\lambda, \nu}=\frac{\nu}{\cosh \left(\nu_{\lambda} H\right)} \int_{-H}^{0} u(z) \cosh \left(\nu_{\lambda}(z+H)\right) d z
$$

By Hölder inequality and by (3.3) it follows that (3.9) is a positive scalar product (see also Lemma 4.1 below). Let us now consider the domain $\mathcal{D}$ of the $\mathcal{C}^{2}$ functions satisfying $u^{\prime}(-H)=0$ and $u(0)=u_{\lambda, \nu}$ and define $(T u)(z)=-u^{\prime \prime}(z)$ for $u \in \mathcal{D}$. Using again (3.3) it is readily verified that $\left(u^{\prime \prime}\right)_{\lambda, \nu}=\nu u^{\prime}(0)+\lambda u(0)$ for every $u \in \mathcal{C}^{2}$. Then we can consider (3.5) as the eigenvalue problem for $T$. As can be readily verified, the operator $T$ is symmetric (with respect to the scalar product (3.9)) and the associated quadratic form

$$
q(u, v)=\int_{-H}^{0} u^{\prime}(z) v^{\prime}(z) d z+\frac{\lambda}{\nu} u(0) v(0)
$$

is positive (actually, it is equivalent to the scalar product of $H^{1}(-H, 0)$ by the Poincaré inequality). By standards results $[1,2]$, for any $\lambda>0$ and $\nu>0$ the functions $\bar{Z}$ and $Z_{n}$ form a complete system, orthogonal with respect to scalar product (3.9). In particular, we have the relations

$$
\int_{-H}^{0} \cos \left(\alpha_{n}(z+H)\right) \cos \left(\alpha_{m}(z+H)\right) d z=\frac{1}{\nu} \cos \left(\alpha_{n} H\right) \cos \left(\alpha_{m} H\right)
$$

for $n \neq m$.

Remark 3.2. By Proposition 3.1, it turns out in particular that a solution to problem $P_{\lambda, \nu}$ is also bounded in the strip $S_{H}$ outside any neighborhood of the interval I. Furthermore, from (3.8) it follows that any solution $\psi \in H^{1}\left(S_{H}\right)$ of problem $P_{\lambda, \nu}$ satisfies the bound

$$
\sup _{|x| \geq R,-H \leq z \leq 0} e^{\sqrt{\lambda+\alpha_{1}^{2}}|x|}|\psi(x, z)|<\infty
$$

for every $R>x_{0}$.

Solutions satisfying (3.11) will be called waveless solutions of problem $P_{\lambda, \nu}$, since the corresponding flow does not produce wave pattern at infinity behind the obstacle (see $[9,13])$. Finally, we remark that such solutions satisfy a further property, which play a crucial role in our proof of the solvability of problem $P_{\lambda, \nu}$ in the following sections.

Proposition 3.2. If $\psi \in H^{1}\left(S_{H}\right)$ solves problem $P_{\lambda, \nu}$, then for any $x$ with $|x|>x_{0}$ the equation

$$
\psi(x, 0)=\frac{\nu}{\cosh \left(\nu_{\lambda} H\right)} \int_{-H}^{0} \psi(x, s) \cosh \left[\nu_{\lambda}(s+H)\right] d s
$$

holds.

The proof follows by Green's theorem, taking account of the boundary conditions and of (3.11) (see also [13, 15]). 


\section{A variational problem for waveless solutions}

In this section we consider a weak formulation of problem $(2.41)-(2.45)$ in a suitable Sobolev-type space contained in $H^{1}\left(S_{H}\right)$. Although a solution of problem $P_{\lambda, \nu}$ does not in general belong to $H^{1}$ (see (3.1)), it turns out that existence (and uniqueness) of variational solutions is the basic result for the proof of the solvability of problem $P_{\lambda, \nu}$ in the next section.

Let us consider the space of the restriction to $S_{H}$ of functions $\chi \in C_{0}^{\infty}\left(\mathbb{R}^{2}\right)$ and make the closure with respect to the norm

$$
\|\chi\|^{2}=\int_{S_{H}}\left(|\nabla \chi|^{2}+|\chi|^{2}\right) d x d z+\int_{F}\left|\chi_{x}\right|^{2} d x .
$$

We denote by $\mathcal{H}\left(S_{H}\right)$ the resulting space. Note that, for the sake of simplicity, we used in the above equation the symbol $\chi_{x}$ to denote the (weak) derivative of the trace $\chi(x, 0)$ on $F$. Actually, the trace of the partial derivative $\chi_{x}$ need not exist for a generic $\chi \in \mathcal{H}\left(S_{H}\right)$. On the other hand, the trace $\chi(x, 0)$ is absolutely continuous on $\bar{F}$ (with square integrable derivative in $F)$ so that it is meaningful to consider the limits $\chi\left( \pm x_{0}, 0\right)$ of $\chi(x, 0)$ when $x \rightarrow x_{0}^{+}$and $x \rightarrow-x_{0}^{-}$, respectively. We will denote by $\mathcal{H}_{0}$ the closed subspace of functions $\chi \in \mathcal{H}$ such that $\chi\left( \pm x_{0}, 0\right)=0$. Assume now that $\mathcal{G} \in L^{2}(I)$ in

(2.42) (more generally, we can take $\mathcal{G} \in H^{-\frac{1}{2}}(I)$, see below). Then multiplication of (2.41) by a test function in $\mathcal{H}_{0}$ and integration by parts taking account of $(2.42)-(2.44)$ lead us to the relation

$$
\int_{S_{H}}(\nabla \psi \cdot \nabla \chi+\lambda \psi \chi) d x d z-\frac{1}{\nu} \int_{F} \psi_{x} \chi_{x} d x=\int_{I} \mathcal{G} \chi d x
$$

For any pair of real constants $\alpha_{ \pm}$let us fix $\psi_{\alpha} \in \mathcal{H}$ such that $\psi_{\alpha}\left( \pm x_{0}, 0\right)=\alpha_{ \pm}$. Then we can state our weak form of problem $P_{\lambda, \nu}$ : that is

Given $\alpha_{ \pm} \in \mathbb{R}$ and $\mathcal{G} \in L^{2}(I)$, find $\psi_{0} \in \mathcal{H}_{0}$ such that $\psi=\psi_{0}+\psi_{\alpha}$ satisfies $(4.2)$,

$$
\begin{aligned}
& \int_{S_{H}}\left(\nabla \psi_{0} \cdot \nabla \chi+\lambda \psi_{0} \chi\right) d x d z-\frac{1}{\nu} \int_{F} \psi_{0 x} \chi_{x} d x \\
& =-\int_{S_{H}}\left(\nabla \psi_{\alpha} \cdot \nabla \chi+\lambda \psi_{\alpha} \chi\right) d x d z+\frac{1}{\nu} \int_{F} \psi_{\alpha x} \chi_{x} d x+\int_{I} \mathcal{G} \chi d x
\end{aligned}
$$

for every $\chi \in \mathcal{H}_{0}$.

It is readily verified that the left-hand side of (4.3) is a continuous bilinear form in $\mathcal{H}\left(S_{H}\right)$ endowed with norm (4.1). Moreover, the right-hand side is clearly a bounded linear functional on $\mathcal{H}_{0}$. However, it is not difficult to see that the bilinear form is not coercive in this space. To overcome this problem, we remark that the a priori condition (3.12) suggests a possible subspace of $\mathcal{H}_{0}$ where coercivity is restored. As it will be clear in the sequel, the delicate point is to check whether a weak solution in this subspace is a (finite energy) solution of problem $P_{\lambda, \nu}$. 
Therefore, we set

$$
V_{0}=\psi \in \mathcal{H}_{0}: \psi(x, 0)=\frac{\nu}{\cosh \nu_{\lambda} H} \int_{-H}^{0} \psi(x, s) \cosh \left[\nu_{\lambda}(s+H)\right] d s \quad\left(|x| \geq x_{0}\right) .
$$

We note that the condition defining the subspace $V_{0}$ is meaningful since, as previously remarked, the trace on $F$ of a function in $\mathcal{H}\left(S_{H}\right)$ is continuous up to the points $\pm x_{0}$. $\mathrm{W}$

We have now

Lemma 4.1. For every pair of constants $\lambda>0$ and $\nu>0$, the bilinear form

$$
a(\psi, \chi)=\int_{S_{H}}(\nabla \psi \cdot \nabla \chi+\lambda \psi \chi) d x d z-\frac{1}{\nu} \int_{F} \psi_{x} \chi_{x} d x
$$

is coercive on $V_{0}$.

Proof. Differentiating the condition in (4.4) we get

$$
\psi_{x}(x, 0)=\frac{\nu}{\cosh \nu_{\lambda} H} \int_{-H}^{0} \psi_{x}(x, s) \cosh \left[\nu_{\lambda}(s+H)\right] d s
$$

which holds in $L^{2}(F)$ for every $\psi \in V_{0}$. By the Hölder inequality, we get

$$
\begin{aligned}
& \frac{1}{\nu} \int_{F}\left|\psi_{x}(x, 0)\right|^{2} d x \\
& \quad \leq \frac{\nu}{\cosh ^{2}\left(\nu_{\lambda} H\right)} \int_{F}\left\{\int_{-H}^{0} \cosh ^{2}\left(\nu_{\lambda}(s+H)\right) d s \int_{-H}^{0}\left|\psi_{x}(x, s)\right|^{2} d s\right\} d x \\
& \quad \leq \frac{1}{2}\left(\frac{\nu H}{\cosh ^{2}\left(\nu_{\lambda} H\right)}+\frac{\nu}{\nu_{\lambda}} \tanh \left(\nu_{\lambda} H\right)\right)\|\nabla \psi\|_{L^{2}\left(S_{H}\right)}^{2} .
\end{aligned}
$$

Eliminating $\nu$ using (3.3) we find the identity

$$
\frac{\nu H}{\cosh ^{2}\left(\nu_{\lambda} H\right)}+\frac{\nu}{\nu_{\lambda}} \tanh \left(\nu_{\lambda} H\right)=\left(1-\frac{\lambda}{\nu_{\lambda}^{2}}\right)\left(1+\frac{2 \nu_{\lambda} H}{\sinh \left(2 \nu_{\lambda} H\right)}\right) .
$$

Thus, the coefficient in the last inequality is strictly less than one for every $\lambda>0$ and $\nu_{\lambda}>0$. Hence, our claim follows

The above result lead us to consider the restriction of (4.3) to the subspace $V_{0}$. Thus, we introduce the following

Definition 4.1. A function $\psi \in \mathcal{H}$ is a solution of the problem $\Pi_{\lambda, \nu}$ if, given a function $\mathcal{G} \in L^{2}(I)$ and a pair of real constants $\alpha_{ \pm}$, the following conditions hold:

a) $\psi$ solves (4.2) for every $\chi \in V_{0}$.

b) $\psi\left( \pm x_{0}, 0\right)=\alpha_{ \pm}$.

c) $\psi$ satisfies the relation

$$
\psi(x, 0)=\frac{\nu}{\cosh \nu_{\lambda} H} \int_{-H}^{0} \psi(x, s) \cosh \left[\nu_{\lambda}(s+H)\right] d s
$$

for every $|x| \geq x_{0}$.

Now, we can prove 
Theorem 4.1. There is a unique solution in $\mathcal{H}$ of problem $\Pi_{\lambda, \nu}$.

Proof. By Lemma 4.1 and the Lax-Milgram theorem, given $\psi_{\alpha} \in \mathcal{H}$ with $\psi_{\alpha}\left( \pm x_{0}, 0\right)=$ $\alpha_{ \pm}$and $\mathcal{G} \in L^{2}(I)$, there is a unique solution $\psi_{0} \in V_{0}$ of equation (4.3), for every $\chi \in V_{0}$. We now show that $\psi_{\alpha}$ can be chosen to satisfy (4.7). Let $\phi_{\alpha} \in H^{1}(\mathbb{R})$ such that $\phi_{\alpha}\left( \pm x_{0}\right)=\alpha_{ \pm}$and let $\eta$ be a smooth function on $[-H, 0]$ satisfying $\eta(0)=1$ and

$$
\frac{\nu}{\cosh \nu_{\lambda} H} \int_{-H}^{0} \eta(s) \cosh \left[\nu_{\lambda}(s+H)\right] d s=1 .
$$

Then, our claim follows by taking $\psi_{\alpha}(x, z)=\phi_{\alpha}(x) \eta(z)$. Now, it is clear that $\tilde{\psi}=$ $\psi_{0}+\psi_{\alpha}$ satisfies (4.2) (for every $\chi \in V_{0}$ ) with the additional conditions $\psi_{\alpha}\left( \pm x_{0}, 0\right)=\alpha_{ \pm}$ and (4.7). Finally, if $\tilde{\psi}$ is another solution of problem $\Pi_{\lambda, \nu}$ with the same data, the function $\psi-\tilde{\psi}$ belongs to $V_{0}$ and solves the homogeneous equation (4.3). Then $\tilde{\psi}=\psi$

The next step is to investigate the properties of a weak solution given by Theorem 4.1 .

Proposition 4.1. Let $\psi \in \mathcal{H}$ be a solution of problem $\Pi_{\lambda, \nu}$. Then $\psi$ satisfies the boundary conditions $(2.42)-(2.44)$ and there exist real constants $\sigma_{ \pm}$such that

$$
(-\Delta+\lambda) \psi(x, z)=\left[\sigma_{+} \delta\left(x-x_{0}\right)+\sigma_{-} \delta\left(x+x_{0}\right)\right] \cosh \left[\nu_{\lambda}(z+H)\right] .
$$

Moreover, $\psi$ satisfies bound (3.11) and therefore it vanishes as $|x| \rightarrow \infty$.

Proof. We first prove (4.8). For this let us consider a test function $\varphi \in \mathcal{D}\left(S_{H}\right)$ satisfying

$$
\int_{-H}^{0} \varphi\left( \pm x_{0}, z\right) \cosh \left[\nu_{\lambda}(z+H)\right] d z=0
$$

and define

$$
\begin{aligned}
& \chi(x, z)= \\
& \begin{cases}\varphi(x, z) & \text { if }|x|<x_{0} \\
\varphi(x, z)+K_{\lambda} \int_{-H}^{0} \varphi(x, s) \cosh \left[\nu_{\lambda}(s+H)\right] d s \cosh \left[\nu_{\lambda}(z+H)\right] & \text { if }|x| \geq x_{0}\end{cases}
\end{aligned}
$$

where

$$
K_{\lambda}=\frac{2}{H}\left\{\frac{\nu_{\lambda}^{2}+\lambda}{\nu_{\lambda}^{2}-\lambda} \frac{\sinh \left(2 \nu_{\lambda} H\right)}{2 \nu_{\lambda} H}-1\right\}^{-1} .
$$

By (4.9) and an elementary calculation, it can be verified that $\chi$ is continuous and belongs to the subspace $V_{0}$ defined in (4.4). Then we get

$$
\begin{aligned}
\int_{S_{H}} & {[\nabla \psi \nabla \chi+\psi \chi] d x d z } \\
\quad= & \frac{1}{\nu} \int_{F} \psi_{x} \chi_{x} d x \\
\quad= & \frac{K_{\lambda}}{\nu} \cosh \left(\nu_{\lambda} H\right) \int_{F} \psi_{x}(x, 0)\left(\int_{-H}^{0} \varphi_{x}(x, s) \cosh \left[\nu_{\lambda}(s+H)\right] d s\right) d x .
\end{aligned}
$$


On the other hand, by direct calculation, using (4.7) and integration by parts with respect to $z$, we also obtain

$$
\begin{aligned}
\int_{S_{H}} & {[\nabla \psi \nabla \chi+\lambda \psi \chi] d x d z } \\
= & \int_{S_{H}}[\nabla \psi \nabla \varphi+\lambda \psi \varphi] d x d z \\
& +\frac{K_{\lambda}}{\nu} \cosh \left(\nu_{\lambda} H\right) \int_{F} \psi_{x}(x, 0)\left(\int_{-H}^{0} \varphi_{x}(x, s) \cosh \left[\nu_{\lambda}(s+H)\right] d s\right) d x \\
& +K_{\lambda}\left[\nu_{\lambda} \sinh \left(\nu_{\lambda} H\right)-\frac{\nu_{\lambda}^{2}}{\nu} \cosh \left(\nu_{\lambda} H\right)+\frac{\lambda}{\nu} \cosh \left(\nu_{\lambda} H\right)\right] \\
\times & \int_{F} \psi(x, 0)\left(\int_{-H}^{0} \varphi(x, s) \cosh \left[\nu_{\lambda}(s+H)\right] d s\right) d x \\
= & \frac{K_{\lambda}}{\nu} \cosh \left(\nu_{\lambda} H\right) \int_{F} \psi_{x}(x, 0)\left(\int_{-H}^{0} \varphi_{x}(x, s) \cosh \left[\nu_{\lambda}(s+H)\right] d s\right) d x
\end{aligned}
$$

where the last identity follows by (3.3). Comparison with (4.10) yields

$$
\int_{S_{H}}[\nabla \psi \nabla \varphi+\lambda \psi \varphi] d x d z=0
$$

for every test function $\varphi$ satisfying (4.9). In particular, $(-\Delta+\lambda) \psi=0$ in $S_{H} \cap\{x \neq$ $\left.\pm x_{0}\right\}$.

Let us now fix $\eta_{0} \in \mathcal{D}\left(S_{H}\right)$ such that $\operatorname{supp} \eta_{0} \subset\left\{x>-x_{0}\right\}$ and

$$
\int_{-H}^{0} \eta_{0}\left(x_{0}, s\right) \cosh \left[\nu_{\lambda}(s+H)\right] d s=1 .
$$

Then for every $\varphi \in \mathcal{D}\left(S_{H} \cap\left\{x>-x_{0}\right\}\right)$ the function

$$
\varphi_{0}(x, z)=\varphi(x, z)-\eta_{0}(x, z) \int_{-H}^{0} \varphi\left(x_{0}, s\right) \cosh \left[\nu_{\lambda}(s+H)\right] d s
$$

belongs to $\mathcal{D}\left(S_{H}\right)$ and satisfies (4.9). Therefore,

$$
\int_{S_{H}}\left[\nabla \psi \nabla \varphi_{0}+\lambda \psi \varphi_{0}\right] d x d z=0 .
$$

Now, by defining

$$
\sigma_{+}=\int_{S_{H}}\left[\nabla \psi \nabla \eta_{0}+\psi \eta_{0}\right] d x d z
$$

we find

$$
\int_{S_{H}}[\nabla \psi \nabla \varphi+\lambda \psi \varphi] d x d z=\sigma_{+} \int_{-H}^{0} \cosh \left[\nu_{\lambda}(s+H)\right] \varphi\left(x_{0}, s\right) d s
$$


for any smooth $\varphi$ with support contained in the half strip $S_{H} \cap\left\{x>-x_{0}\right\}$. This statement and the analogous one for functions with support in $S_{H} \cap\left\{x<x_{0}\right\}$ are readily shown to be equivalent to (4.8).

We now check boundary conditions $(2.42)$ - (2.44). To this aim we remark that every restriction to $\bar{S}_{H}$ of a function $\chi \in \mathcal{D}\left(\mathbb{R}^{2}\right)$ with support non-intersecting the lines $x= \pm x_{0}$ can be represented in the form $\chi=\chi_{0}+\varphi_{1}$ on $\bar{S}_{H}$ where $\chi_{0} \in V_{0}$ and $\varphi_{1}$ is a smooth function with support in $S_{H} \cap\left\{x \neq x_{0}\right\}$. In fact, this follows by setting

$$
\varphi_{1}(x, z)=\left\{\int_{-H}^{0} \chi(x, s) \cosh \left[\nu_{\lambda}(s+H)\right] d s-\frac{\cosh \nu_{\lambda} H}{\nu} \chi(x, 0)\right\} \zeta(z)
$$

where $\zeta \in \mathcal{D}(-H, 0)$ satisfies $\int_{-H}^{0} \zeta(s) \cosh \left[\nu_{\lambda}(s+H)\right] d s=1$. Then, by (4.11) and (4.2) we obtain

$$
\begin{aligned}
\int_{S_{H}}[\nabla \psi \nabla \chi+\lambda \psi \chi] d x d z & =\int_{S_{H}}\left[\nabla \psi \nabla \chi_{0}+\lambda \psi \chi_{0}\right] d x d z \\
& =\frac{1}{\nu} \int_{F} \psi_{x} \chi_{0 x} d x+\int_{\mathcal{G}} \chi_{0} d x \\
& =\frac{1}{\nu} \int_{F} \psi_{x} \chi_{x} d x+\int_{\mathcal{G}} \chi d x
\end{aligned}
$$

for every smooth $\chi$ with support in $\bar{S}_{H} \cap\left\{x \neq x_{0}\right\}$. Now boundary conditions (2.42) (2.44) follow by standard arguments.

Finally, since $\psi$ is in $\mathcal{H}$ and satisfies (3.1) for $|x|>x_{0}$, the conclusions of Remark 3.2 apply so that $\psi$ satisfies $(3.11)$

The following result is crucial for the discussion of the next section.

Proposition 4.2. Given a function $\mathcal{G} \in L^{2}(I)$ and a pair of real constants $\alpha_{ \pm}$, there is a unique function $\psi \in \mathcal{H}\left(S_{H}\right)$ satisfying equation (4.8), boundary conditions $(2.42)-(2.44)$ and additional condition (2.45). Moreover, if $\mathcal{G} \in H^{1 / 2}(I)$ in condition $(2.42)$, the trace $\psi(x, 0)$ has continuous derivative on $F$ up to the points $\left( \pm x_{0}, 0\right)$, and the constants $\sigma_{ \pm}$in equation (4.8) are given by

$$
\begin{aligned}
& \frac{\sigma_{+}-\sigma_{-}}{2} \sin \left(\mu_{\lambda} x_{0}\right) \\
& =C\left(\nu_{\lambda}\right)\left\{\int_{-x_{0}}^{x_{0}}\left[\nu_{\lambda} \sinh \left(\nu_{\lambda} H\right) \psi(x, 0)-\cosh \left(\nu_{\lambda} H\right) \mathcal{G}(x)\right] \sin \left(\mu_{\lambda} x\right) d x\right. \\
& \quad+\frac{\mu_{\lambda}}{\nu} \cos \left(\mu_{\lambda} x_{0}\right) \cosh \left(\nu_{\lambda} H\right)\left(\alpha_{+}-\alpha_{-}\right) \\
& \left.\quad-\frac{1}{\nu} \sin \left(\mu_{\lambda} x_{0}\right) \cosh \left(\nu_{\lambda} H\right)\left[\psi_{x}\left(x_{0}^{+}, 0\right)+\psi_{x}\left(-x_{0}^{-}, 0\right)\right]\right\}
\end{aligned}
$$

and

$$
\begin{aligned}
& \frac{\sigma_{+}+\sigma_{-}}{2} \cos \left(\mu_{\lambda} x_{0}\right) \\
& \quad=C\left(\nu_{\lambda}\right)\left\{\int_{-x_{0}}^{x_{0}}\left[\nu_{\lambda} \sinh \left(\nu_{\lambda} H\right) \psi(x, 0)-\cosh \left(\nu_{\lambda} H\right) \mathcal{G}(x)\right] \cos \left(\mu_{\lambda} x\right) d x\right.
\end{aligned}
$$




$$
\begin{aligned}
& -\frac{\mu_{\lambda}}{\nu} \sin \left(\mu_{\lambda} x_{0}\right) \cosh \left(\nu_{\lambda} H\right)\left(\alpha_{+}+\alpha_{-}\right) \\
& \left.-\frac{1}{\nu} \cos \left(\mu_{\lambda} x_{0}\right) \cosh \left(\nu_{\lambda} H\right)\left[\psi_{x}\left(x_{0}^{+}, 0\right)-\psi_{x}\left(-x_{0}^{-}, 0\right)\right]\right\}
\end{aligned}
$$

where

$$
C\left(\nu_{\lambda}\right)=\nu_{\lambda}\left[\nu_{\lambda} H+\sinh \left(\nu_{\lambda} H\right) \cosh \left(\nu_{\lambda} h\right)\right]^{-1} .
$$

Proof. We note that for any function $\psi$ satisfying equation (4.8) one has

$$
\begin{aligned}
& \int_{S_{H}} \chi(-\Delta+\lambda) \psi d x d z \\
& \quad=\sigma_{+} \int_{-H}^{0} \chi\left(x_{0}, z\right) \cosh \left[\nu_{\lambda}(z+H)\right] d z+\sigma_{-} \int_{-H}^{0} \chi\left(-x_{0}, z\right) \cosh \left[\nu_{\lambda}(z+H)\right] d z \\
& \quad=\frac{\cosh \left(\nu_{\lambda} H\right)}{\nu}\left[\sigma_{+} \chi\left(x_{0}, 0\right)+\sigma_{-} \chi\left(-x_{0}, 0\right)\right] \\
& \quad=0
\end{aligned}
$$

for every $\chi \in V_{0}$. Assume now that $\psi^{(1)}$ satisfies the same boundary conditions as $\psi$ and solves equation (4.8) (with constants $\sigma_{ \pm}^{(1)}$ ). Then integration by parts in the previous relation shows that $\psi-\psi^{(1)}$ is a solution of the homogeneous problem $\Pi_{\lambda, \nu}$. Hence, $\psi^{(1)}=\psi$.

Let us now define the function

$$
\begin{gathered}
\phi(x, z)=\psi(x, z)+\frac{\sigma_{+}}{\mu_{\lambda}} \theta\left(x-x_{0}\right) \sin \left[\mu_{\lambda}\left(x-x_{0}\right)\right] \cosh \left[\nu_{\lambda}(z+H)\right] \\
-\frac{\sigma_{-}}{\mu_{\lambda}} \theta\left(-x-x_{0}\right) \sin \left[\mu_{\lambda}\left(x+x_{0}\right)\right] \cosh \left[\nu_{\lambda}(z+H)\right] .
\end{gathered}
$$

By direct computation and by (4.8) we get $(-\Delta+\lambda) \phi(x, z)=0$ in $S_{H}$. Moreover, $\phi$ belongs to $H_{l o c}^{1}\left(S_{H}\right)$ and satisfies the same boundary conditions as $\psi$. Suppose now that $\mathcal{G} \in H^{\frac{1}{2}}(I)$ and consider a small neighborhood $\mathcal{B}$ of the point $\left(x_{0}, 0\right)$ in $\bar{S}_{H}$. Defining $\mathcal{I}=\partial \mathcal{B} \cap\{z=0\}$, we can regard $\mathcal{I}$ as a polygonal boundary with angle $\omega=\pi$ at $\left(x_{0}, 0\right)$. Then the function $\phi$ satisfies

$$
\begin{aligned}
\Delta \phi(x, z)=\lambda \phi(x, z) & \in H^{1}(\mathcal{B}) \\
\phi_{z}(x, 0)=\mathcal{G}(x) & \in H^{\frac{1}{2}}(\mathcal{I} \cap I) \\
\phi_{x x}(x, 0)=-\nu \phi_{z}(x, 0) & \in H^{-\frac{1}{2}}(\mathcal{I} \cap F) .
\end{aligned}
$$

Now we can apply regularity arguments (see [14] and references therein) relying on known results for elliptic problems in polygonal domains $[4,5]$ and conclude that

$$
\phi(x, z)-C r^{\frac{1}{2}} \cos \frac{\theta}{2} \in H^{2}(\mathcal{B})
$$

where $C$ is a constant and $(r, \theta)$ equal the polar coordinates around the point, with $\theta=0$ on $I$ and $\theta=\pi$ on $F$. In particular, one gets from (4.16)

$$
\phi_{z}(x, z)-C r^{-\frac{1}{2}} \sin \frac{\theta}{2} \in H^{1}(\mathcal{B}) .
$$


Letting $\theta \rightarrow \pi$ we find that $\phi_{z}(x, 0)-C\left|x-x_{0}\right|^{-\frac{1}{2}}$ belongs to $H^{\frac{1}{2}}(\mathcal{I} \cap F)$. Using again the boundary condition on $F$ we conclude that

$$
\phi_{x}(x, 0)-C\left|x-x_{0}\right|^{\frac{1}{2}} \in H^{\frac{3}{2}}(\mathcal{I} \cap F) .
$$

Obviously, an analogous result holds in a neighborhood of $\left(-x_{0}, 0\right)$. Hence, we have the continuity of $\phi_{x}(\cdot, 0)$ on $\bar{F}$. Recalling $(4.15)$, the same is true for $\psi_{x}(\cdot, 0)$.

Finally, let $Q_{0}=\left(-x_{0}, x_{0}\right) \times(-H, 0)$ and consider the functions

$$
\begin{aligned}
& \bar{\gamma}^{1}(x, z)=\cos \left(\mu_{\lambda} x\right) \cosh \left[\nu_{\lambda}(z+H)\right] \\
& \bar{\gamma}^{2}(x, z)=\sin \left(\mu_{\lambda} x\right) \cosh \left[\nu_{\lambda}(z+H)\right] .
\end{aligned}
$$

Since $\psi$ and $\bar{\gamma}^{i}(i=1,2)$ satisfy $(3.1)$ in $Q_{0}$, we can apply Green's theorem and obtain

$$
\int_{\partial Q_{0}}\left[\psi \frac{\partial \bar{\gamma}^{i}}{\partial n}-\bar{\gamma}^{i} \frac{\partial \psi}{\partial n}\right] d l=0
$$

where $d l$ is the line element on the boundary of $Q_{0}$. We can now explicitly evaluate (4.17) using the boundary conditions and observing that (4.15) and the regularity of $\phi$ in $S_{H}$ imply

$$
\lim _{\varepsilon \rightarrow 0}\left[\psi_{x}\left( \pm x_{0}+\varepsilon, z\right)-\psi_{x}\left( \pm x_{0}-\varepsilon, z\right)\right]=-\sigma_{ \pm} \cosh \left[\nu_{\lambda}(z+H)\right]
$$

for every $z \in(-H, 0)$. Then by elementary calculations and taking account of (4.7) we obtain $(4.13)-(4.14)$

Remark 4.1. From the uniqueness result of the above proposition, it follows that problem $P_{\lambda, \nu}$ has a solution in $H^{1}\left(S_{H}\right)$ (waveless solutions, see Remark 3.2) if and only if $\sigma_{+}=\sigma_{-}=0$. Moreover, the solution is uniquely determined by $\mathcal{G}$ and $\alpha_{ \pm}$.

As it is clear from the previous proof, the function $\phi$ defined in (4.15) satisfies the requirements of problem $P_{\lambda, \nu}$ except asymptotic condition (2.46). Now, in order to establish existence (and uniqueness) of problem $P_{\lambda, \nu}$, we will proceed as follows: first, we prove that there exists a non-trivial, two-dimensional subspace of $H_{l o c}^{1}\left(S_{H}\right)$ made of solutions of homogeneous problem (2.41) - (2.45). Then we show that a solution of problem $P_{\lambda, \nu}$ can be obtained by adding to $\phi$ a suitable function in the above space, if certain compatibility conditions hold. 


\section{Existence and uniqueness of the solution to problem $\boldsymbol{P}_{\lambda, \nu}$}

To begin with, we introduce two special solutions of problem $\Pi_{\lambda, \nu}$ : We denote by $\psi_{s}$ the solution to problem $\Pi_{\lambda, \nu}$ with $\alpha_{ \pm}= \pm \alpha_{s}= \pm \sin \left(\mu_{\lambda} x_{0}\right)$ and

$$
\mathcal{G}(x)=\nu_{\lambda} \tanh \left(\nu_{\lambda} H\right) \sin \left(\mu_{\lambda} x\right) .
$$

Similarly, $\psi_{c}$ is the solution to problem $\Pi_{\lambda, \nu}$ with $\alpha_{ \pm}=\alpha_{c}=\cos \left(\mu_{\lambda} x_{0}\right)$ and

$$
\mathcal{G}(x)=\nu_{\lambda} \tanh \left(\nu_{\lambda} H\right) \cos \left(\mu_{\lambda} x\right) .
$$

Then, $\psi_{s}$ and $\psi_{c}$ satisfy (2.42) - (2.45) with the above data in conditions (2.42), (2.45). Moreover, by symmetry properties of the data and by uniqueness, we have $\psi_{s}(-x, z)=$ $-\psi_{s}(x, z)$ and $\psi_{c}(-x, z)=\psi_{c}(x, z)$, so that $\psi_{s}$ and $\psi_{c}$ satisfy $(4.8)$ with $\sigma_{+}=-\sigma_{-} \equiv \sigma_{s}$ and $\sigma_{+}=\sigma_{-} \equiv \sigma_{c}$, respectively. Finally, the regularity properties of Proposition 4.2 hold and from (4.13) - (4.14) we get the relations

$$
\begin{aligned}
\sigma_{s} \sin \left(\mu_{\lambda} x_{0}\right)= & C\left(\nu_{\lambda}\right)\left\{\int_{-x_{0}}^{x_{0}} \nu_{\lambda} \sinh \left(\nu_{\lambda} H\right)\left[\psi_{s}(x, 0)-\sin \left(\mu_{\lambda} x\right)\right] \sin \left(\mu_{\lambda} x\right) d x\right. \\
& \left.+\frac{2}{\nu}\left[\mu_{\lambda} \cos \left(\mu_{\lambda} x_{0}\right)-\psi_{s x}\left(x_{0}^{+}, 0\right)\right] \sin \left(\mu_{\lambda} x_{0}\right) \cosh \left(\nu_{\lambda} H\right)\right\}
\end{aligned}
$$

and

$$
\begin{aligned}
\sigma_{c} \cos \left(\mu_{\lambda} x_{0}\right)= & C\left(\nu_{\lambda}\right)\left\{\int_{-x_{0}}^{x_{0}} \nu_{\lambda} \sinh \left(\nu_{\lambda} H\right)\left[\psi_{c}(x, 0)-\cos \left(\mu_{\lambda} x\right)\right] \cos \left(\mu_{\lambda} x\right) d x\right. \\
& \left.-\frac{2}{\nu}\left[\mu_{\lambda} \sin \left(\mu_{\lambda} x_{0}\right)+\psi_{c x}\left(x_{0}^{+}, 0\right)\right] \cos \left(\mu_{\lambda} x_{0}\right) \cosh \left(\nu_{\lambda} H\right)\right\}
\end{aligned}
$$

where $C\left(\nu_{\lambda}\right)$ is the same as in Proposition 4.2.

Remark 5.1. It is worthwhile to remark that the functions $\psi_{s}, \psi_{c}$ and the constants $\sigma_{s}, \sigma_{c}$ depend only on $x_{0}, H$ and $\nu$. Moreover, the pairs $\psi_{s}, \sigma_{s}$ and $\psi_{c}, \sigma_{c}$ can be explicitly evaluated for special values of the parameter $\mu_{\lambda}$. In fact, let us choose $\mu_{\lambda}=n \frac{\pi}{x_{0}} \quad(n \geq 1)$ and set

$$
\psi_{s}(x, z)= \begin{cases}\frac{1}{\cosh \left(\nu_{\lambda} H\right)} \sin \left(\mu_{\lambda} x\right) \cosh \left[\nu_{\lambda}(z+H)\right] & \text { if }|x|<x_{0} \\ 0 & \text { if }|x| \geq x_{0} .\end{cases}
$$

Similarly, for $\mu_{\lambda}=\left(n-\frac{1}{2}\right) \frac{\pi}{x_{0}} \quad(n \geq 1)$ we define

$$
\psi_{c}(x, z)= \begin{cases}\frac{1}{\cosh \left(\nu_{\lambda} H\right)} \sin \left(\mu_{\lambda} x\right) \cosh \left[\nu_{\lambda}(z+H)\right] & \text { if }|x|<x_{0} \\ 0 & \text { if }|x| \geq x_{0} .\end{cases}
$$

Then it is readily verified by direct calculations that both these functions belong to $\mathcal{H}$ and verify the required boundary conditions and equation (4.8). The corresponding values of $\sigma_{s}$ and $\sigma_{c}$ are

$$
\begin{gathered}
\sigma_{s}\left(n \frac{\pi}{x_{0}}\right)=(-1)^{n} \frac{n \frac{\pi}{x_{0}}}{\cosh \left(n H \frac{\pi}{x_{0}}\right)} \\
\sigma_{c}\left(\left(n-\frac{1}{2}\right) \frac{\pi}{x_{0}}\right)=(-1)^{n} \frac{\left(n-\frac{1}{2}\right) \frac{\pi}{x_{0}}}{\cosh \left(\left(n-\frac{1}{2}\right) H \frac{\pi}{x_{0}}\right)}
\end{gathered}
$$


where $n \geq 1$. Then, our claim follows by Proposition 4.2.

Now, following (4.15) we define

$$
\begin{aligned}
\phi_{s}(x, z)= & \psi_{s}(x, z) \\
& +\frac{\sigma_{s}}{\mu_{\lambda}}\left[\theta\left(x-x_{0}\right) \sin \left[\mu_{\lambda}\left(x-x_{0}\right)\right]+\theta\left(-x-x_{0}\right) \sin \left[\mu_{\lambda}\left(x+x_{0}\right)\right]\right] \\
& \times \cosh \left[\nu_{\lambda}(z+H)\right]
\end{aligned}
$$

and

$$
\begin{aligned}
\phi_{c}(x, z)= & \psi_{c}(x, z) \\
& +\frac{\sigma_{c}}{\mu_{\lambda}}\left[\theta\left(x-x_{0}\right) \sin \left[\mu_{\lambda}\left(x-x_{0}\right)\right]-\theta\left(-x-x_{0}\right) \sin \left[\mu_{\lambda}\left(x+x_{0}\right)\right]\right] \\
& \times \cosh \left[\nu_{\lambda}(z+H)\right] .
\end{aligned}
$$

Then the functions

$$
\begin{aligned}
\zeta_{s}(x, z) & =\phi_{s}(x, z)-\sin \left(\mu_{\lambda} x\right) \frac{\cosh \left(\left[\nu_{\lambda}(z+H)\right]\right.}{\cosh \left(\nu_{\lambda} H\right)} \\
\zeta_{c} & =\phi_{c}(x, z)-\cos \left(\mu_{\lambda} x\right) \frac{\cosh \left(\left[\nu_{\lambda}(z+H)\right]\right.}{\cosh \left(\nu_{\lambda} H\right)}
\end{aligned}
$$

solve the homogeneous problem (2.41) - (2.44) and satisfy

$$
\zeta_{s}\left( \pm x_{0}, 0\right)=\zeta_{c}\left( \pm x_{0}, 0\right)=0
$$

By exploiting the asymptotic behaviour of the functions $\zeta_{s}$ and $\zeta_{c}$ we will obtain a condition of unique solvability to problem $P_{\lambda, \nu}$. To this aim, the next lemma is crucial:

Lemma 5.1. The representations

$$
\begin{aligned}
& \zeta_{s}(x, z)=\left[\mathcal{A}_{s} \sin \left(\mu_{\lambda} x\right) \pm \mathcal{B}_{s} \cos \left(\mu_{\lambda} x\right)\right] \cosh \left[\nu_{\lambda}(z+H)\right]+\zeta_{0}^{s}(x, z) \\
& \zeta_{c}(x, z)=\left[ \pm \mathcal{A}_{c} \sin \left(\mu_{\lambda} x\right)+\mathcal{B}_{c} \cos \left(\mu_{\lambda} x\right)\right] \cosh \left[\nu_{\lambda}(z+H)\right]+\zeta_{0}^{c}(x, z)
\end{aligned}
$$

hold for $\pm x>x_{0}$ where $\zeta_{0}^{s}$ and $\zeta_{0}^{c}$ are rapidly decreasing as $|x| \rightarrow \infty$ and

$$
\begin{array}{ll}
\mathcal{A}_{s}=\frac{\sigma_{s}}{\mu_{\lambda}} \cos \left(\mu_{\lambda} x_{0}\right)-\frac{1}{\cosh \left(\nu_{\lambda} H\right)} & \mathcal{B}_{s}=-\frac{\sigma_{s}}{\mu_{\lambda}} \sin \left(\mu_{\lambda} x_{0}\right) \\
\mathcal{A}_{c}=\frac{\sigma_{c}}{\mu_{\lambda}} \cos \left(\mu_{\lambda} x_{0}\right) & \mathcal{B}_{c}=-\frac{\sigma_{c}}{\mu_{\lambda}} \sin \left(\mu_{\lambda} x_{0}\right)-\frac{1}{\cosh \left(\nu_{\lambda} H\right)} .
\end{array}
$$

Proof. It follows by elementary calculations from (5.5) - (5.6) and recalling the asymptotic properties of $\psi_{s}$ and $\psi_{c}$

We can now state our condition of unique solvability: 
Proposition 5.1. Let $\lambda>0$ and $\nu>0$ be given and let $\mu_{\lambda}=\sqrt{\nu_{\lambda}^{2}-\lambda}$, where $\nu_{\lambda}$ is the solution of $\tanh \left(\nu_{\lambda} H\right)=\frac{1}{\nu}\left(\nu_{\lambda}-\frac{\lambda}{\nu_{\lambda}}\right)$. Assume that the condition

$$
\sigma_{s} \cos \left(\mu_{\lambda} x_{0}\right)-\sigma_{c} \sin \left(\mu_{\lambda} x_{0}\right) \neq \frac{\mu_{\lambda}}{\cosh \left(\nu_{\lambda} H\right)}
$$

holds, where $\sigma_{s}$ and $\sigma_{c}$ satisfy $(5.1)-(5.2)$. Then problem $P_{\lambda, \nu}$ is uniquely solvable.

Proof. We first prove uniqueness of the solution. For this, assume that $\psi_{0}$ is a solution to problem $P_{\lambda, \nu}$ with $\mathcal{G}=0$ and that $\psi_{0}\left( \pm x_{0}, 0\right)=0$. Let $\mathcal{A}_{0}$ and $\mathcal{B}_{0}$ be the coefficients of the oscillating terms in the asymptotic expansion (3.1) for $\psi_{0}$. We apply Green's formula to $\psi_{0}$ and to each of the functions $\zeta^{s}$ and $\zeta^{c}$ defined by (5.3) - (5.4) in the bounded rectangle $(-R, R) \times(-H, 0)$, with $R>x_{0}$. Then, taking account of Lemma 5.1, we get

$$
\begin{aligned}
0= & \lim _{R \rightarrow \infty} \int_{\partial Q_{R}}\left[\zeta^{s} \partial_{n} \psi_{0}-\psi_{0} \partial_{n} \zeta^{s}\right] d l \\
= & \lim _{R \rightarrow \infty}\left\{\int_{\left[-R,-x_{0}\right] \cup\left[x_{0}, R\right]}\left[\zeta^{s}(x, 0) \psi_{0, z}(x, 0)-\psi_{0}(x, 0) \zeta_{z}^{s}(x, 0)\right] d x\right. \\
& \left.+\int_{-H}^{0}\left[\zeta^{s}(R, z) \psi_{0, x}(R, z)-\psi_{0}(R, z) \zeta_{x}^{s}(R, z)\right] d z\right\} \\
= & \lim _{R \rightarrow \infty}\left\{-\frac{1}{\nu} \int_{\left[-R,-x_{0}\right] \cup\left[x_{0}, R\right]}\left[\zeta^{s}(x, 0) \psi_{0, x x}(x, 0)-\psi_{0}(x, 0) \zeta_{x x}^{s}(x, 0)\right] d x\right. \\
& \left.+\int_{-H}^{0}\left[\zeta^{s}(R, z) \psi_{0, x}(R, z)-\psi_{0}(R, z) \zeta_{x}^{s}(R, z)\right] d z\right\} \\
= & \lim _{R \rightarrow \infty}\left\{-\frac{1}{\nu}\left[\zeta^{s}(R, 0) \psi_{0, x}(R, 0)-\psi_{0}(R, 0) \zeta_{x}^{s}(R, 0)\right]\right. \\
& \left.+\int_{-H}^{0}\left[\zeta^{s}(R, z) \psi_{0, x}(R, z)-\psi_{0}(R, z) \zeta_{x}^{s}(R, z)\right] d z\right\} .
\end{aligned}
$$

Explicit calculation of the last term using representations (3.8) and (5.8) lead us to the relation

$$
\begin{aligned}
& \mu_{\lambda}\left\{\frac{1}{\nu} \cosh ^{2}\left(\nu_{\lambda} H\right)-\frac{1}{2}\left[H+\frac{\cosh \left(\nu_{\lambda} H\right) \sinh \left(\nu_{\lambda} H\right)}{\nu_{\lambda}}\right]\right\}\left[\mathcal{A}_{s} \mathcal{B}_{0}-\mathcal{A}_{0} \mathcal{B}_{s}\right] \\
& =\frac{\mu_{\lambda} \cosh ^{2}\left(\nu_{\lambda} H\right)}{\nu}\left\{1-\frac{1}{2}\left(1-\frac{\lambda}{\nu_{\lambda}^{2}}\right)\left(1+\frac{2 \nu_{\lambda} H}{\sinh \left(2 \nu_{\lambda} H\right)}\right)\right\}\left[\mathcal{A}_{s} \mathcal{B}_{0}-\mathcal{A}_{0} \mathcal{B}_{s}\right]=0 .
\end{aligned}
$$

Clearly, the above equation is equivalent to

$$
\mathcal{A}_{s} \mathcal{B}_{0}-\mathcal{A}_{0} \mathcal{B}_{s}=0
$$

An analogous calculation with $\zeta_{c}$ and $\psi_{0}$ gives

$$
\mathcal{A}_{c} \mathcal{B}_{0}-\mathcal{A}_{0} \mathcal{B}_{c}=0
$$


System (5.13) - (5.14) is equivalent to $\mathcal{A}_{0}=\mathcal{B}_{0}=0$ if and only if

$$
\mathcal{A}_{s} \mathcal{B}_{c}-\mathcal{A}_{c} \mathcal{B}_{s} \neq 0
$$

By (5.10) this is equivalent to (5.11). In this case, $\psi_{0}$ is a solution in $H^{1}\left(S_{H}\right)$ of the homogeneous problem $P_{\lambda, \nu}$. Then $\psi_{0}=0$ by Remark 4.1 .

We now show that the same condition (5.11) guarantees the existence of a solution. Take $\psi$ as in Proposition 4.2 and consider the function $\phi$ defined by (4.15). For every pair of real constants $\gamma$ and $\delta$ we set

$$
\psi^{(\gamma, \delta)}=\phi-\gamma \zeta^{s}-\delta \zeta^{c}
$$

where $\zeta^{s}$ and $\zeta^{c}$ are defined by (5.5) - (5.6). Then by Lemma 5.1 and recalling the properties of $\phi$ one verifies that $\psi^{(\gamma, \delta)}$ satisfies all the relations of problem $P_{\lambda, \nu}$, including asymptotic condition (2.46) if the pair $(\gamma, \delta)$ solves the linear system

$$
\begin{gathered}
\gamma \mathcal{A}_{s}-\delta \mathcal{A}_{c}=\frac{-\sigma_{-}}{\mu_{\lambda}} \cos \left(\mu_{\lambda} x_{0}\right) \\
\gamma \mathcal{B}_{s}-\delta \mathcal{B}_{c}=\frac{\sigma_{-}}{\mu_{\lambda}} \sin \left(\mu_{\lambda} x_{0}\right) .
\end{gathered}
$$

Clearly, this system admits a (unique) solution if and only if (5.11) holds

We now investigate the validity of condition (5.11) as $\mu_{\lambda}$ varies in the interval $(0,+\infty)$. By (5.3) - (5.4) we see that (5.11) fails for $\mu_{\lambda}=k \frac{\pi}{2 x_{0}} \quad(k \geq 1)$, that is for $\nu_{\lambda}=\sqrt{\lambda+\left(k \frac{\pi}{2 x_{0}}\right)^{2}}$. We can prove that there are no other singular values of $\mu_{\lambda}\left(\right.$ or $\left.\nu_{\lambda}\right)$.

Lemma 5.2. For every $\nu_{\lambda}$ and $\mu_{\lambda}$ as in Proposition 5.1, the relation

$$
\sigma_{s} \cos \left(\mu_{\lambda} x_{0}\right)-\sigma_{c} \sin \left(\mu_{\lambda} x_{0}\right)=\frac{\mu_{\lambda}}{\cosh \left(\nu_{\lambda} H\right)}+D\left(\nu_{\lambda}\right) \sin \left(\mu_{\lambda} x_{0}\right) \cos \left(\mu_{\lambda} x_{0}\right)
$$

holds where $D\left(\nu_{\lambda}\right)>0$.

The proof is given in the appendix of [15] and is similar to the proof of an analogous result [14: Proposition 4.8] for the case $\lambda=0$. We remark here that the crucial point of the proof is the variational characterization of the weak solutions $\psi_{s}$ and $\psi_{c}$ defined in Section 5 as the minimum points of the functionals representing the coefficients $\sigma_{s}$ and $\sigma_{c}$, respectively.

Theorem 5.1. Let $\lambda>0$ and $\nu>0$ be a pair of constants and let $\mu_{\lambda}=\sqrt{\nu_{\lambda}^{2}-\lambda}$, where $\nu_{\lambda}$ is the positive solution of $\tanh \left(\nu_{\lambda} H\right)=\frac{1}{\nu}\left(\nu_{\lambda}-\frac{\lambda}{\nu_{\lambda}}\right)$. Assume that

$$
\mu_{\lambda} \neq k \frac{\pi}{2 x_{0}} \quad(k \geq 1)
$$

Then, for every $\mathcal{G} \in L^{2}(I)$ and $\alpha_{ \pm} \in \mathbb{R}$, problem $P_{\lambda, \nu}$ is uniquely solvable. Moreover, if $\mathcal{G} \in H^{\frac{1}{2}}(I)$, the solution $\psi$ is continuous and bounded in the closed strip $\bar{S}_{H}$, and the trace $\psi(x, 0) \quad\left(|x|>x_{0}\right)$ has continuous derivative up to the points $\left( \pm x_{0}, 0\right)$.

Proof. Assuming (5.18), unique solvability of problem $P_{\lambda, \nu}$ follows by Proposition 5.1 and by Lemma 5.2 . If $\mathcal{G} \in H^{\frac{1}{2}}(I)$ in condition $(2.42)$, the regularity properties of the solution can be readily verified from representation (5.16) and by the second part of the proof of Proposition 4.2 


\section{The solvability of the three-dimensional problem}

In this final section, we discuss the solvability of the three-dimensional problem $P$ (see Section 2) in terms of Fourier expansion (2.33). We will also make some comments on the physical interpretation of the results obtained. We point out that (2.33) solves problem $P$ if one can differentiate the series term by term and if each coefficient $\psi_{m}$ is a solution of problem $P_{\lambda_{m}}(m \geq 0)$, with $\lambda_{m}=\frac{m^{2} \pi^{2}}{L^{2}}$. Now, from Theorem 5.1 and by elementary calculations (recalling that $\nu=\frac{g}{c^{2}}$ ) we find that problem $P_{\lambda_{m}}$ is solvable for every $m \geq 1$, provided $c \neq c_{m, k} \quad(k \geq 1)$ where

$$
c_{m, k}=\frac{2 x_{0}}{k}\left\{\frac{g}{\pi}\left(\frac{m^{2}}{L^{2}}+\frac{k^{2}}{4 x_{0}^{2}}\right)^{\frac{1}{2}} \tanh \left[\pi H\left(\frac{m^{2}}{L^{2}}+\frac{k^{2}}{4 x_{0}^{2}}\right)^{\frac{1}{2}}\right]\right\}^{\frac{1}{2}} .
$$

Moreover, the solution $\psi_{m}$ depends on two parameters fixing the values of $\psi_{m}\left( \pm x_{0}, 0\right)$ and is continuous in a closed, two-dimensional strip, if the boundary datum in (2.37) belongs to $H^{\frac{1}{2}}\left(\tilde{x}_{-}, \tilde{x}_{+}\right)$.

Remark 6.1. Note that the right-hand side of (6.1) depends on the geometric features of problem $P$ : the depth $H$ and the width $L$ of the domain $\Omega_{0}$, and the length of the interval $\left(-x_{0}, x_{0}\right)$. We further remark that $(6.1)$ provides the correct "singular values" of the velocity also for $m=0$ (see [14]). In this case we have $c_{0, k} \leq \sqrt{g H}$ for $k \geq 1$ so that the singular velocities are all subcritical.

Thus, if $c \neq c_{m, k}$ for every $m \geq 0$ and $k \geq 1$, we are left with the proof of suitable estimates on the functions $\psi_{m}$, allowing differentiation term by term of series (2.33). By the results of the previous sections, these estimates will depend on the functions $\mathcal{F}_{m}$ (which are determined by the shape of the obstacle's surface, see (2.34)) and by the values of $\psi_{m}\left( \pm x_{0}, 0\right)$ with $m \geq 1$.

For the sake of brevity, we will not discuss the problem in this general setting and consider only those functions $\mathcal{F}$ with finite number of terms different from zero in expansion (2.34). Then, it is natural to require the additional condition $\psi_{m}\left( \pm x_{0}, 0\right)=0$ for every $m$ such that $\mathcal{F}_{m}^{\prime}=0$. In this way, by Theorem 5.1 , we have $\psi_{m}=0$ for the same $m$ so that also series (2.33) has a finite number of terms different from zero. Clearly, in this case we get solutions of problem $P$, which however still depend on a finite number of parameters (fixing the values of the perturbed potential $\Psi$ on the lines $\left.x= \pm x_{0}, z=0,0 \leq y \leq L\right)$. We conjecture that this arbitrariness could be eliminated by looking for best regular solutions. Actually, recalling the results of Sections 4 and 5, every solution $\psi$ of problem $P_{\lambda, \nu}$ with datum $\mathcal{G} \in H^{\frac{1}{2}}(I)$ in condition (2.42) satisfies

$$
\psi-C_{+} \eta_{+}-C_{-} \eta_{-} \in H^{2}\left(S_{H}\right)
$$

where $C_{ \pm}$are constants and $\eta_{ \pm}$are two functions defined in the strip $S_{H}$ and vanishing respectively outside a neighborhood of $\left( \pm x_{0}, 0\right)$. Moreover, by exploiting the boundary conditions as in the proof of Proposition 4.12, it can be shown that every solution in $H^{2}\left(S_{H}\right)$ (and with smooth enough boundary datum on the interval $I$ ) has bounded and continuous derivatives up to the boundary of $S_{H}$. Hence, it is an interesting open problem to find out if the values of $\psi\left( \pm x_{0}, 0\right)$ can be fixed in such a way that $C_{+}=$ 
$C_{-}=0$ in (6.2). In that case, we could conclude that the solution with everywhere bounded velocity field is uniquely determined.

We make a final comment on the interpretation of the condition $c \neq c_{m, k}$ from the point of view of the nonlinear problem discussed in Section 2. We note that, in the case $m=0$, relation (6.1) gives the values of the critical velocities for the existence of twodimensional water waves (in fluid without obstacles) bifurcating from the trivial parallel flow and with wave lengths $\lambda_{k} \approx 4 \frac{x_{0}}{k}$ (see [18: Chapter 71]). On the other hand, by our linearization assumptions (see Section 2) the boundary length of any longitudinal section of the surface-piercing obstacle converges to the limit $D=2 x_{0}$ for $\varepsilon \rightarrow 0$. Then we get the values $2 \frac{D}{k}$ for the wave lengths at the bifurcation points $c_{0, k}$. Thus, the condition $c \neq c_{0, k}$ appears as a "non-resonance condition" between the limit length of the obstacle and the gravitational waves bifurcating from the free parallel flow at the same velocity. For $m>0$ we have a similar relation involving the dimensions of the obstacle at $\varepsilon=0$ (i.e. in the limit of a plane, rectangular domain) and the linear steady waves in the channel. We stress that values (6.1) should not be considered as singular velocities for the exact nonlinear problem. They rather suggest that, fixing $c>0$ and letting $\varepsilon \rightarrow 0$ in the equations of the nonlinear problem, the lines of contact between the free surface and the obstacle cannot approach arbitrary limit positions if the assumptions of Section 2 hold.

\section{References}

[1] Brezis, H.: Analyse fonctionnelle - Théorie et applications. Paris: Masson 1983.

[2] Dautray, R. and J. L. Lions: Mathematical Analysis and Numerical Methods for Science and Technology, Vol. 2. Berlin - Heidelberg: Springer-Verlag 1988.

[3] Do, C.: Le probleme spectral associe au modele de Neumann-Kelvin. Portugaliae Math. 41 (1982), $211-231$.

[4] Grisvard, G.: Elliptic Problems in Nonsmooth Domains (Pitman Adv. Publ. Program). London: Pitman 1985.

[5] Grisvard, P.: Singularities in Boundary Value Problems (Research Notes in Applied Mathematics: Vol. 22). Paris: Masson 1992.

[6] Kuznetsov, N. G., Maz'ya, V. G. and V. Vainberg: Linear Water Waves. Cambridge: Cambridge Univ. Press 2002.

[7] Kuznetsov, N. G. and V. G. Maz'ya: On Unique solvability of the plane Neumann-Kelvin problem. Math. USSR - Sbornik 63 (1989), 425 - 446.

[8] Kuznetsov, N. G. and V. G. Maz'ya: On a well-posed formulation of the two-dimensional plane Neumann-Kelvin problem for a surface-piercing body. In: Current Problems of Analysis and Mathematical Physics, Taormina 1998. Rome: Aracne 2000, pp. 77 - 109.

[9] Kuznetsov, N. G. and O. Motygin: On the waveless statement of the two-dimensional Neumann-Kelvin problem for a surface-piercing body. IMA J. Appl. Math. 59 (1997), 25 -42 .

[10] Livshits, M. and V. G. Maz'ya: Solvability of the two-dimensional Kelvin-Neumann problem for a submerged circular cylinder. Appl. Anal. 64 (1997), 1 - 5.

[11] Newman, J. N.: Marine Hydrodynamics. Cambridge: MIT Press 1977. 
[12] Pagani, C. D. and D. Pierotti: The forward motion of an unsymmetric surface-piercing cylinder: the solvability of the nonlinear problem in the supercritical case. Quart. J. Mecc. Appl. Math. 54 (2000), $1-22$.

[13] Pierotti, D.: The subcritical motion of a surface-piercing cylinder: existence and regularity of waveless solutions of the linearized problem. Adv. Diff. Equ. 7 (2002), 385 - 418.

[14] Pierotti, D.: On unique solvability and regularity in the linearized two dimensional wave resistance problem. Quarterly Appl. Math. (to appear).

[15] Pierotti, D. and S. Gatti: The three-dimensional flow in a channel past a semisubmerged obstacle: solvability of a linearized problem. Politecnico di Milano 495/P (2002).

[16] Stoker, J. J.: Water Waves. New York: Intersci. 1957.

[17] Ursell, F.: Mathematical notes on the two-dimensional Kelvin-Neumann problem. In: Proc. Thirteenth Sympos. Naval Hydrodynamics, Tokio 1980. Shipbuilding Res. Assoc. Japan 1981, pp. $145-151$.

[18] Zeidler, E.: Nonlinear Functional Analysis and its Applications. Volume IV: Applications to Mathematical Physics. New York: Springer-Verlag 1988.

Received 22.07.2002; in revised form 09.01.2003 